\title{
Research Article \\ Clinical Outcome and Predictors of Intestinal Obstruction Surgery in Ethiopia: A Cross-Sectional Study
}

\author{
Tesfaye Derseh, ${ }^{1}$ Tariku Dingeta, ${ }^{2}$ Mohammed Yusouf, ${ }^{1}$ and Binyam Minuye $\mathbb{D}^{3}$ \\ ${ }^{1}$ Department of Obstetrics and Gynecology, College of Medicine and Health Science, Harar, Ethiopia \\ ${ }^{2}$ School of Public Health, College of Medicine and Health Sciences, Haramaya University, Harar, Ethiopia \\ ${ }^{3}$ College of Health Sciences, Debretabor University, Debretabor, Ethiopia
}

Correspondence should be addressed to Binyam Minuye; biniamminuye@yahoo.com

Received 17 August 2020; Revised 13 November 2020; Accepted 16 November 2020; Published 23 November 2020

Academic Editor: Gernot Kaiser

Copyright ( $) 2020$ Tesfaye Derseh et al. This is an open access article distributed under the Creative Commons Attribution License, which permits unrestricted use, distribution, and reproduction in any medium, provided the original work is properly cited.

Background. Despite the advancement in the healthcare system, the impact of surgical interventions on public health systems will continue to grow. But predicting the outcome is challenging. Concerns related to unexpected outcomes and delays in the diagnosis of postoperative complications are the major issue. Intestinal obstruction is a common life-threatening surgical condition followed by fatal and nonfatal postoperative complications. This study was aimed at assessing results after surgery for intestinal obstruction in a hospital of Ethiopia. Methodology. An institutional-based cross-sectional study was conducted among 254 postoperative patients admitted with intestinal obstruction from January 1, 2014, to December 31, 2017. Data were coded and entered into EpiData 4.2.0.0 software and exported to the Statistical Package for the Social Sciences version 22 for analysis. A binary logistic regression model was used for analysis. All variables with a $p$ value $<0.25$ during bivariable analysis were considered for multivariable logistic regression analysis. Results. The magnitude of poor surgical outcome of intestinal obstruction was $21.3 \%$ for patients enrolled into this investigation. The age group of $\geq 55$ years (adjusted odds ratio $(\mathrm{AOR})=2.9,95 \% \mathrm{CI}: 1.03,8.4)$, duration of illness of $\geq 24 \mathrm{hrs}$ ( $\mathrm{AOR}=3.1,95 \% \mathrm{CI}: 1.03,9.4)$, preoperative diagnosis of a gangrenous large bowel $(\mathrm{AOR}=3.6$, $95 \% \mathrm{CI}: 1.3,9.8$ ), and a gangrenous small bowel ( $\mathrm{AOR}=4.2,95 \% \mathrm{CI}: 1.3,13.7)$ were significantly associated with poor surgical outcome. Conclusions. The magnitude of poor surgical outcome was high. Age, late presentation of illness, and gangrenous bowel obstructions were significantly associated with poor outcomes. So, concern should be given in early detection and followup of patients who came late and older patients.

\section{Background}

Each year, millions of people undertake surgical interventions which account for an estimated $13 \%$ of the world's total disability-adjusted life years, $0.5-5 \%$ crude mortality rate, and $25 \%$ postoperative complications [1]. Intestinal obstruction is one of the surgical emergencies caused by a blockage in the flow of intestinal contents $[2,3]$. It increases morbidity and mortality $[4,5]$. The burden of intestinal obstruction in Ethiopia ranges from $21.8 \%$ to $4.6 \%[6,7]$.

Despite the advancements in the field of medicine, introduction of a safe surgery checklist, improved monitoring and related safety practices during anesthesia, surgical technique, and conservative management, the surgical management outcome of intestinal obstruction remains a challenge to the healthcare system [1]. Surgical care is followed by fatal and nonfatal postoperative complications from the diseases itself, the operation, and the anesthesia [8]. Globally, the World Health Organization (WHO) 2019 fact sheet on healthcareassociated infections revealed that a hundred million patients were affected by healthcare-associated infections, each year. Point prevalence ranges from 3.5 to $12 \%$ in developed and 5.7 to $19.1 \%$ in low- and middle-income countries [9]. The burden of healthcare-associated infections was also reported in sub-Saharan Africa [10], Botswana (13.4\%) [11], South Africa (8\%) [12], and Ethiopia (13-35.8\%) [13-16].

Universally, intestinal obstruction varies from country to country in terms of incidence and management outcomes depending on ethnicity, age group, dietary habits, residence, geographic location, the living condition of the community, 
presentation, length of hospital stay, comorbid illness, duration of operation, duration of illness, presence of peritonitis, and service provision [5, 17-19]. Difficulties in using the checklist, postoperative intra-abdominal infections, the inadequacy of training, and insufficient amount of anesthesiologists, nurses, and support staff [19-22] were some of the challenges which lead to poor management outcomes.

In this case, few studies were conducted in north and central Ethiopia referral hospitals related to the pattern of admissions $[6,23]$; there is a paucity of research on predictors of surgical outcome of intestinal obstruction in Ethiopia, particularly in regional hospitals. Thus, this study was conducted to assess clinical outcomes and predictors of intestinal obstruction surgery in Chiro General Hospital, Eastern Ethiopia.

\section{Methods and Materials}

2.1. Study Design, Period, Setting, and Population. An institutional-based cross-sectional study was conducted in Chiro General Hospital, Eastern Ethiopia. Chiro Town is situated at $328 \mathrm{~km}$ to the east of Addis Ababa. The hospital provided healthcare service for more than 1,441,008 populations in its catchment area with a total of 166 beds. All patients surgically treated for intestinal obstruction from January 1, 2014, to December 31, 2017, were the study populations. A total of 254 patients suffering from intestinal obstruction were included in the study.

2.2. Data Collection Methods. Data were collected based on a structured data abstraction sheet from medical records and registers. The abstraction sheet includes sociodemographic factors, type of procedure, and duration of illness. The data was extracted from medical charts. The data was collected by $3 \mathrm{BSc}$ nurses and $1 \mathrm{MSc}$ clinical midwifery supervisor. Completeness of each recording format was checked before collecting the data.

\subsection{Variables}

2.3.1. Dependent Variable. The surgical management outcome is considered the dependent variable (poor, good).

2.3.2. Independent Variables. Sociodemographic characteristics (age, sex, and residence), duration of illness, cause of obstruction, procedure done, and intraoperative findings are the independent variables.

2.3.3. Operational Definitions. Surgical treatment means surgical exploration of the abdomen which is determined by the nature of obstruction [1].

Poor management outcome is the condition of the patient after the procedure has been done where the patient develops postoperative complications (dehiscence, surgical site infection, pneumonia, and shock) or died until the patient is discharged from the hospital [2].

2.3.4. Data Quality Control. The pretest was done on 5\% of the sample size in Felege Hiwot Referral Hospital. One-day training was given for data collectors and supervisors on data collection tools and data collection procedures. Supervision and completeness of each abstraction sheet had
TABLE 1: Sociodemographic distribution of patients surgically treated for intestinal obstruction in Chiro General Hospital, 2018.

\begin{tabular}{lcc}
\hline Category & Frequency & Percent \\
\hline Age & 222 & 87 \\
$<55$ & 32 & 13 \\
$\geq 55$ & & \\
Sex & 226 & 89 \\
Male & 28 & 11 \\
Female & & \\
Residence & 56 & 22 \\
Chiro & 198 & 78 \\
Out of Chiro & & \\
\hline
\end{tabular}

been checked by the principal investigator and the supervisors on a daily basis. Checking for double data entry was done by two data clerks, and the consistency of the entered data was cross-checked.

2.3.5. Data Processing and Analysis. Data were entered, coded, cleaned, and checked by EpiData statistical software version 4.2.0.0, and analysis was done using SPSS version 22 statistical software. Descriptive statistics was presented using tables, figures, and texts. Binary logistic regression was used for analysis. During bivariable analysis, seven variables with a $p$ value $<0.25$ were considered for multivariable logistic regression analysis. The odds ratio along with $95 \%$ CI was estimated to identify factors associated with the outcome variable. The level of significance was declared at a $p$ value $\leq 0.05$.

\section{Results}

3.1. Sociodemographic Characteristics of Study Participants. A total of two hundred fifty-four patients participated in the study. The mean age of the participants was 34 years (SD $\pm 16.24)$. The majority $(226,89 \%)$ were males (Table 1$)$.

3.2. Clinical Presentation, Duration, and Preoperative Diagnosis of Intestinal Obstruction. All patients presented with the clinical symptoms of abdominal pain, whereas 245 (96.6\%), 242 (95.3\%), and 232 (91.3\%) patients present with vomiting, abdominal distension, and failure to pass flatus and feces, respectively. In addition, $8.7 \%$ of patients had a history of groin swelling. $65.7 \%$ were diagnosed with simple small bowel obstruction (SBO). On the other hand, 13.4\% were diagnosed as having simple large bowel obstruction (LBO) (Figure 1).

3.3. Intraoperative Finding and Surgical Procedures Done. Almost half of admissions (47.6\%) were due to small bowel volvulus, followed by $16.5 \%$ adhesion and bands and $13.8 \%$ sigmoid volvulus. Derotation and decompression (DD) and resection and anastomosis (RA) surgical procedures were done for $42.1 \%$ and $29.5 \%$ of patients, respectively (Table 2).

3.4. Magnitude of Poor Management Outcome. The magnitude of poor surgical management outcome of intestinal obstruction was $21.3 \%$ (95\% CI: $16.5-26.4)$. More than half (55.5\%) had wound site infection (hematoma and incisional 


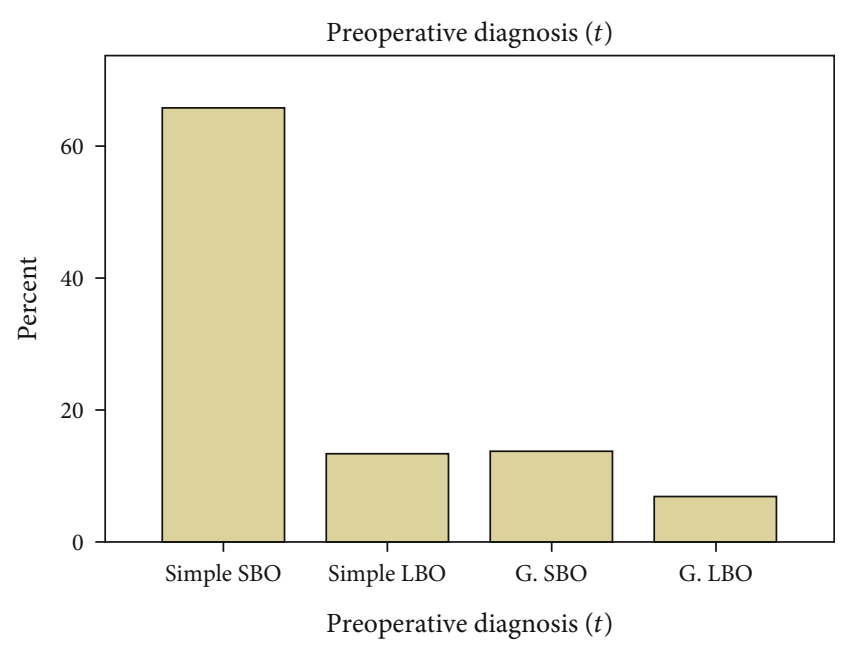

FIgURE 1: Clinical diagnosis of surgically treated patients with intestinal obstruction in Chiro General Hospital, $2018(N=254)$. SBO: small bowl obstruction; LBO: large bowl obstruction; G: gangrenous.

TABLE 2: Intraoperative finding and surgical procedures done for patients with IO who were treated surgically in Chiro General Hospital, $2018(N=254)$.

\begin{tabular}{lcc}
\hline Variables & Frequency & Percent (\%) \\
\hline Intraoperative finding & & \\
Small bowel volvulus & 212 & 47.6 \\
Adhesion and bands & 42 & 16.5 \\
Sigmoid volvulus & 35 & 13.8 \\
Intussusception & 22 & 8.7 \\
Strangulated hernia & 21 & 8.3 \\
Others & 13 & 5.1 \\
Type of procedures done & & \\
Derotation and decompression & 107 & 42.1 \\
Resection and anastomosis & 75 & 29.5 \\
Adhesiolysis \& band release & 38 & 15.0 \\
Herniorrhaphy & 13 & 5.1 \\
Hartmann's colostomy & 10 & 3.9 \\
Reduction & 9 & 3.5 \\
Other procedures & 6 & 2.4 \\
\hline
\end{tabular}

surgical site infection), $14.8 \%$ postoperative pneumonia, and $11.1 \%$ anastomotic leak.

3.5. Factors Associated with Poor Management Outcome. A binary logistic regression was done to identify the association between the poor outcome of intestinal obstruction and independent variables. In the bivariable analysis, age $\geq 55$ years, out of Chiro residence, duration of illness $\geq 24 \mathrm{hrs}$, preoperative diagnosis of gangrenous SBO and gangrenous $\mathrm{LBO}$, the operative finding of gangrenous small bowel volvulus, and the operative procedure of DD and RA were identified. However, in multiple logistic regression analysis, the age group of $\geq 55$ years, duration of illness of $\geq 24$ hours, preoper- ative diagnosis of gangrenous $\mathrm{SBO}$, and gangrenous $\mathrm{LBO}$ were significantly associated with poor surgical outcomes.

Patients with the age of $\geq 55$ years were nearly 3 times more likely to develop poor outcomes as compared with patients whose age was $\leq 55$ years $(\mathrm{AOR}=2.9,95 \% \mathrm{CI}: 1.03$, 8.4). Patients who came late ( $\geq 24$ hours) were about three times more likely to develop poor outcomes compared with patients who came early ( $<24$ hours) $(\mathrm{AOR}=3.1,95 \% \mathrm{CI}$ : $1.03,9.4)$. Those patients with gangrenous $\mathrm{LBO}$ and gangrenous SBO had, respectively, 3.6 and 4.2 times higher odds of developing unfavorable outcome than patients with simple SBO $(\mathrm{AOR}=3.6,95 \% \mathrm{CI}: 1.3,9.8$ and $\mathrm{AOR}=4.2,95 \% \mathrm{CI}$ : $1.3,13.7$, respectively) (Table 3 ).

\section{Discussion}

Intestinal obstruction is the surgical emergency followed by fatal and nonfatal postoperative complications. While surgical intervention is intended to save the lives of individuals, unsafe surgical care can cause substantial harm to the patient.

The magnitude of poor management outcomes of intestinal obstruction was $21.3 \%$. This study is in line with the study done in Adama (24.6\%) [6] and India (25.89\%) [24]. But the magnitude in this study is lower than those in studies conducted in Canada (64\%) [25] and Nigeria (66.5\%) [26]. This might be due to differences in the cause, type of procedure done, and study population. Intussusception was the most common cause of intestinal obstruction in Nigeria. On the contrary, it was higher than the study done in Kenya (13.6\%) [27]. The possible reason might be due to the difference in the place of residence. In Kenya, $58.7 \%$ of patients came from rural dwellers [27], whereas $78 \%$ in the current study. It is believed that patients who came from the urban area could have good awareness on the importance of getting health service earlier. The other possible reason might be the difference in the standard of surgical procedures.

In the current study, old age, late presentation of illness, and preoperative diagnosis of a gangrenous bowel were significantly associated with the occurrence of poor outcomes. Surgical site infection threatens the lives of millions of patients each year and contributes to the spread of antibiotic resistance bacteria (Staphylococcus aureus, Escherichia coli, and Pseudomonas aeruginosa $[28,29]$. In the current study, wound site infection (hematoma and incisional SSI) was found to be the major poor surgical outcome $(55.5 \%)$. In addition, postoperative pneumonia and anastomotic leak were reported among $14.8 \%$ and $11.1 \%$ patients, respectively. Similarly, SSI was reported in Adama, Kenya, Botswana, and Nigeria which accounts for $39.3 \%, 33 \%, 9 \%$, and $31.4 \%$, respectively $[6,26-28]$. The incidence of SSI can be reduced by administering perioperative antibiotics such as ampicillin, cefotaxime, metronidazole, and amoxicillin/clavulanate. Literature showed that patients with an acute abdomen should receive preoperative antibiotics and postoperative antibiotics in case of perforation [30,31]. One study showed no significant effect on the postoperative outcome by administering metronidazole for perforated appendicitis [32]. Perioperative antibiotic administrations depend on different factors such as anatomic region undergoing the specific 
TABLE 3: Factors associated with poor surgical management outcomes of intestinal obstruction surgery in patients admitted to CGH, 2018.

\begin{tabular}{|c|c|c|c|c|}
\hline \multirow{2}{*}{ Variables } & \multicolumn{2}{|c|}{ Surgical outcome } & \multirow{2}{*}{ COR: $95 \% \mathrm{CI}$} & \multirow{2}{*}{ COR: $95 \% \mathrm{CI}$} \\
\hline & Poor $(\%)$ & Good (\%) & & \\
\hline \multicolumn{5}{|l|}{ Age } \\
\hline$\geq 55$ & $12(37.5)$ & $20(62.5)$ & $2.6(1.2-5.7)$ & $2.9(1.03-8.4)^{*}$ \\
\hline$<55$ & $42(18.9)$ & $180(81.1)$ & 1 & 1 \\
\hline \multicolumn{5}{|l|}{ Residence } \\
\hline Out of Chiro & $48(24.2)$ & $150(75.8)$ & $2.7(1.1-6.6)$ & $2.7(0.9-7.6)$ \\
\hline Chiro & $6(10.7)$ & $50(89.3)$ & 1 & 1 \\
\hline \multicolumn{5}{|l|}{ Duration of illness } \\
\hline$\geq 24$ hours & $49(28.3)$ & $124(71.7)$ & $6(2.3-15.7)$ & $3.1(1.03-9.4)^{*}$ \\
\hline$<24$ hours & $5(6.2)$ & $76(93.8)$ & 1 & 1 \\
\hline \multicolumn{5}{|c|}{ Preoperative diagnosis of IO } \\
\hline Simple LBO & $6(17.6)$ & $28(82.4)$ & $1.7(0.6-4.6)$ & $1.5(0.5-4.5)$ \\
\hline Gangrenous SBO & $21(60.0)$ & $14(40.0)$ & $11.7(5.1-26.7)$ & $3.6(1.3-9.8)^{*}$ \\
\hline Gangrenous LBO & $8(44.4)$ & $10(55.6)$ & $6.2(2.2-17.7)$ & $4.2(1.3-13.7)^{*}$ \\
\hline Simple SBO & $19(11.4)$ & $148(88.6)$ & 1 & 1 \\
\hline \multicolumn{5}{|c|}{ Intraoperative procedure done } \\
\hline $\mathrm{DD}$ & $9(8.4)$ & $98(91.6)$ & $0.2(0.1-0.5)$ & $0.7(0.3-1.9)$ \\
\hline Other procedures & $45(30.6)$ & $102(69.4)$ & 1 & 1 \\
\hline RA & $34(45.3)$ & $41(54.7)$ & $6.6(3.4-12.6)$ & $2.0(0.8-5.3)$ \\
\hline Other procedures & $20(11.2)$ & $159(88.8)$ & 1 & 1 \\
\hline \multicolumn{5}{|l|}{ Intraoperative finding } \\
\hline Gangrenous SBV & $19(59.4)$ & $13(40.6)$ & $7.8(3.5-17.2)$ & $2.1(0.7-6.2)$ \\
\hline Other findings & $35(15.8)$ & $187(84.2)$ & 1 & 1 \\
\hline
\end{tabular}

*Significant at a $p$ value $<0.05 ; 1$ is the reference. $\mathrm{SBO}=$ small bowel obstruction; $\mathrm{LBO}=$ large bowel obstruction; $\mathrm{DD}=$ derotation and decompression; $\mathrm{RA}=$ resection and anastomosis; $\mathrm{SBV}=$ small bowel volvulus; $\mathrm{SV}=$ sigmoid volvulus.

surgical procedure, timing of surgery, age of the patient, time of antibiotic administration, urgency of the procedure, and availability of the drugs. Surgical antibiotic administration after incision was associated with a significantly higher incidence of SSI compared with administration before incision $[33,34]$. So, the burden of SSI can be minimized by applying WHO recommendations [35].

Patients aged $\geq 55$ years were more likely to develop poor management outcomes compared with those patients whose ages are less than 55 years. The study is in line with studies done in Gondar [17], Japan [36], and China [37]. This is true, as age increases the physiologic process of organs and tissue progressively degenerates over time [38] and decreased immune response [39].

Patients who came late were more likely to develop poor outcomes than patients who came earlier. This is consistent with studies conducted in Adama [6] and Gondar [17]. This might be due to poor health-seeking behavior and a poor transportation system in this subregion. Late presentation in the case of intestinal obstruction accounts for disastrous outcomes, notably a high rate of complications, long hospital stay, and high mortality rates [40]. Identifying which patient needs early surgery is difficult, given the lack of specific clinical or radiographic signs [41]. Moreover, clinical presentation of surgical problems in the elderly may be subtle, and handling stress poorly because of physiological change may lead to delay in diagnosis.

\section{Conclusion}

The magnitude of poor management outcomes was high. Old age, late presentation of illness, and gangrenous bowel obstructions were significantly associated with poor surgical outcome. Emphasis should be given in improving the patient's outcome using a surgical patient safety checklist and creating awareness in seeking care for emergency conditions and postoperative complications. In addition, effective infection prevention activities have to be implemented in the hospital setting. Future research should be done on barriers of delay to take care for surgical illnesses in a prospective manner by including variables such as educational status, occupational status, income, and knowledge-related factors.

\section{Abbreviations}

AOR: Adjusted odds ratio

CGH: Chiro General Hospital

COR: Crude odds ratio

CHMS: College of Health and Medical Sciences

GLBO: Gangrenous large bowel obstruction

GSBO: Gangrenous small bowel obstruction

HU: Haramaya University

IHRERC: Institutional Health Research Ethics Review Committee

IO: Intestinal obstruction 
LBO: Large bowel obstruction

RA: Resection and anastomosis

SBO: $\quad$ Small bowel obstruction

SBV: $\quad$ Small bowel volvulus

SOP: $\quad$ Standard of procedure

SSI: $\quad$ Surgical site incision/infection.

\section{Data Availability}

All relevant data are within the manuscript.

\section{Ethical Approval}

Ethical clearance was obtained from the Institutional Health Research Ethics Review Committee (IHRERC), College of Health and Medical Sciences, Haramaya University. A permission letter was obtained from Chiro General Hospital, and data were collected from medical records.

\section{Conflicts of Interest}

The authors declare that they have no competing interests.

\section{Authors' Contributions}

TD1 worked on designing the study, training the data collectors, supervising the data collectors, interpreting the result, and preparing the manuscript. TD1, TD, MY, and BM analyzed and interpreted the result. BM wrote the manuscript. All authors are involved starting from design and data interpretation to critically reviewing the manuscript.

\section{Acknowledgments}

The authors acknowledge the data collectors and administrative staff of Chiro General Hospital.

\section{References}

[1] WHO, "Patient safety and safe surgery," 2020, https://www .who.int/patientsafety/safesurgery/en/.

[2] R. Collins, "Hamilton Bailey's emergency surgery," Annals of the Royal College of Surgeons of England, vol. 85, no. 3, 2003.

[3] B. Trilling, E. Girard, P. Waroquet, and C. Arvieux, "Intestinal obstruction, an overview," Revue de L'infirmière, vol. 217, pp. 16-18, 2016.

[4] E. Ojo, C. Ihezue, A. Sule, O. Ismaila, A. Dauda, and A. Adejumo, "Aetiology, clinical pattern and outcome of adult intestinal obstruction in JOS, north central Nigeria," African Journal of Medicine and Medical Sciences, vol. 43, Supplement 1, pp. 29-36, 2014.

[5] M. Kapan, A. Onder, S. Polat et al., "Mechanical bowel obstruction and related risk factors on morbidity and mortality," Journal of Current Surgery, vol. 2, no. 2, pp. 55-61, 2012.

[6] U. Soressa, A. Mamo, D. Hiko, and N. Fentahun, "Prevalence, causes and management outcome of intestinal obstruction in Adama Hospital, Ethiopia," BMC Surgery, vol. 16, no. 1, p. 38, 2016.

[7] G. Tamrat, M. Osman, N. Deyessa, M. Taye, R. Lett, and A. Bekele, "Delay of emergency surgical interventions in Ethi- opia: patient and health system factors," East and Central African Journal of Surgery, vol. 23, no. 2, pp. 59-65, 2018.

[8] S. W. Bickler, T. G. Weiser, N. Kassebaum et al., "Global burden of surgical conditions," in Essential Surgery: Disease Control Priorities, World Bank Group Eliberary, 2015.

[9] World Health Organization, Health Care-Associated Infections: Fact Sheet, WHO, Geneva, 2020.

[10] C. Rothe, C. Schlaich, and S. Thompson, "Healthcare-associated infections in sub-Saharan Africa," Journal of Hospital Infection, vol. 85, no. 4, pp. 257-267, 2013.

[11] P. Mpinda-Joseph, B. D. Anand Paramadhas, G. Reyes et al., "Healthcare-associated infections including neonatal bloodstream infections in a leading tertiary hospital in Botswana," Hospital Practice, vol. 47, no. 4, pp. 203-210, 2019.

[12] A. Nair, W. Steinberg, T. Habib, H. Saeed, and J. Raubenheimer, "Prevalence of healthcare-associated infection at a tertiary hospital in the Northern Cape Province, South Africa," South African Family Practice, vol. 60, no. 5, pp. 162-167, 2018.

[13] W. W. Yallew, A. K. Takele, and F. M. Yehuala, "Point prevalence of hospital-acquired infections in two teaching hospitals of Amhara region in Ethiopia," Drug, Healthcare and Patient Safety, vol. Volume 8, pp. 71-76, 2016.

[14] S. Ali, M. Birhane, S. Bekele et al., "Healthcare associated infection and its risk factors among patients admitted to a tertiary hospital in Ethiopia: longitudinal study," Antimicrobial Resistance \& Infection Control, vol. 7, no. 1, 2018.

[15] T. Alemayehu, E. Tadesse, S. Ayalew et al., "High burden of nosocomial infections caused by multi-drug resistant pathogens in pediatric patients at Hawassa University Comprehensive Specialized Hospital," Ethiopian Medical Journal, vol. 58, 2019.

[16] N. Endalafer, S. Gebre-Selassie, and B. Kotiso, "Nosocomial bacterial infections in a tertiary hospital in Ethiopia," Journal of Infection Prevention, vol. 12, no. 1, pp. 38-43, 2011.

[17] S. Tsegaye, M. Osman, and A. Bekele, "Surgically treated acute abdomen at Gondar University Hospital, Ethiopia," East and Central African Journal of Surgery, vol. 12, no. 1, pp. 53-57, 2007.

[18] D. J. Kacey, "Maingot's abdominal operations," Journal of the American Medical Association, vol. 300, no. 7, pp. 852-853, 2008.

[19] D. Shida, K. Tagawa, K. Inada et al., "Modified enhanced recovery after surgery (ERAS) protocols for patients with obstructive colorectal cancer," BMC Surgery, vol. 17, no. 1, pp. 1-6, 2017.

[20] K. L. Close, L. S. Baxter, V. A. Ravelojaona et al., "Overcoming challenges in implementing the WHO Surgical Safety Checklist: lessons learnt from using a checklist training course to facilitate rapid scale up in Madagascar," BMJ Global Health, vol. 2, article e000430, Suppl 4, 2017.

[21] M. Tokunaga, Y. Tanizawa, E. Bando, T. Kawamura, and M. Terashima, "Poor survival rate in patients with postoperative intra-abdominal infectious complications following curative gastrectomy for gastric cancer," Annals of Surgical Oncology, vol. 20, no. 5, pp. 1575-1583, 2013.

[22] C. M. Kelly, N. Starr, N. P. Raykar, R. R. Yorlets, C. Liu, and M. Derbew, "Provision of surgical care in Ethiopia: challenges and solutions," Global Public Health, vol. 13, no. 11, pp. 16911701, 2017.

[23] B. Kotiso and Z. Abdurahman, "Pattern of acute abdomen in adult patients in Tikur Anbessa Teaching Hospital, Addis 
Ababa, Ethiopia," East and Central African Journal of Surgery, vol. 12, no. 1, pp. 47-52, 2007.

[24] A. Souvik, M. Z. Hossein, D. Amitabha, M. Nilanjan, and R. Udipta, "Etiology and outcome of acute intestinal obstruction: a review of 367 patients in Eastern India," Saudi Journal of Gastroenterology, vol. 16, no. 4, pp. 285-287, 2010.

[25] J. E. Springer, J. G. Bailey, P. J. Davis, and P. M. Johnson, "Management and outcomes of small bowel obstruction in older adult patients: a prospective cohort study," Canadian Journal of Surgery, vol. 57, no. 6, pp. 379-384, 2014.

[26] A. O. Bankole, A. O. Osinowo, and A. A. Adesanya, "Predictive factors of management outcome in adult patients with mechanical intestinal obstruction," Nigerian Postgraduate Medical Journal, vol. 24, no. 4, pp. 217-223, 2017.

[27] P. B. Ooko, B. Sirera, S. Saruni, H. M. Topazian, and R. White, "Pattern of adult intestinal obstruction at Tenwek hospital, in south-western Kenya," Pan African Medical Journal, vol. 20, no. 1, 2015.

[28] J. C. Mwita, S. Souda, M. G. M. D. Magafu, A. Massele, B. Godman, and M. Mwandri, "Prophylactic antibiotics to prevent surgical site infections in Botswana: findings and implications," Hospital Practice, vol. 46, no. 3, pp. 97-102, 2018.

[29] W. Metwally and R. Aamir, "Prevalence and antimicrobial resistance patterns of nosocomial pathogens causing surgical site infections in an Egyptian University Hospital," Microbes and Infectious Diseases, vol. 1, no. 3, pp. 226-237, 2020.

[30] K. Daskalakis, C. Juhlin, and L. Påhlman, “The use of pre- or postoperative antibiotics in surgery for appendicitis: a systematic review," Scandinavian Journal of Surgery, vol. 103, no. 1, pp. 14-20, 2014.

[31] B. A. Coakley, E. S. Sussman, T. S. Wolfson et al., "Postoperative antibiotics correlate with worse outcomes after appendectomy for nonperforated appendicitis," Journal of the American College of Surgeons, vol. 213, no. 6, pp. 778-783, 2011.

[32] Q. Shang, Q. Geng, X. Zhang, and C. Guo, "The efficacy of combined therapy with metronidazole and broad-spectrum antibiotics on postoperative outcomes for pediatric patients with perforated appendicitis," Medicine, vol. 96, no. 47, article e8849, 2017.

[33] M. T. Hawn, J. S. Richman, C. C. Vick et al., "Timing of surgical antibiotic prophylaxis and the risk of surgical site infection," JAMA Surgery, vol. 148, no. 7, pp. 649-657, 2013.

[34] M. T. Hawn, K. M. Itani, S. H. Gray, C. C. Vick, W. Henderson, and T. K. Houston, "Association of timely administration of prophylactic antibiotics for major surgical procedures and surgical site infection," Journal of the American College of Surgeons, vol. 206, no. 5, pp. 814-819, 2008.

[35] B. Allegranzi, P. Bischoff, S. de Jonge et al., "New WHO recommendations on preoperative measures for surgical site infection prevention: an evidence-based global perspective," The Lancet Infectious Diseases, vol. 16, no. 12, pp. e276-e287, 2016.

[36] K. Morikane, H. Honda, and S. Suzuki, "Factors associated with surgical site infection following gastric surgery in Japan," Infection Control \& Hospital Epidemiology, vol. 37, no. 10, pp. 1167-1172, 2016.

[37] H.-l. Chen, M.-q. Zhou, W. Tian, K.-x. Meng, and H.-f. He, "Effect of age on breast cancer patient prognoses: a population-based study using the SEER 18 database," PLoS One, vol. 11, no. 10, article e0165409, 2016.
[38] C. Strøm and L. S. Rasmussen, "Challenges in anaesthesia for elderly," Singapore Dental Journal, vol. 35, pp. 23-29, 2014.

[39] D. L. Woodland and M. A. Blackman, "Immunity and age: living in the past?," Trends in Immunology, vol. 27, no. 7, pp. 303-307, 2006.

[40] G. Ntakiyiruta and B. Mukarugwiro, "The Patterns of intestinal obstruction at Kibogola Hospital, rural hospital in Rwanda," East and Central African Journal of Surgery, vol. 14, no. 2, pp. 103-108, 2009.

[41] N. Kulvatunyou, V. Pandit, S. Moutamn et al., "A multiinstitution prospective observational study of small bowel obstruction: clinical and computerized tomography predictors of which patients may require early surgery," Journal of Trauma and Acute Care Surgery, vol. 79, no. 3, pp. 393-398, 2015. 